\title{
Isothermal brain predicting Mcculloch-Pitts logic implicating Walter freeman ionic diffusion and Lotfi Zadeh fuzzy logic that might be useful for coding autonomous vehicles to be coexisting with the pedestrians
}

\begin{abstract}
We wish to demonstrate mathematically a possibility of fuzzy thinking in homo sapiens. We begin with the fundamental physiological fact that human brains are kept at a constant temperature, we can prove McCulloch-Pitts sigmoid- $\sigma$ formula adopted by Artificial Neural Networks (ANN) as neuronal firing rates. Then, we verify sigmoid- $\sigma$ to be the exact solution of nonlinear Riccati differential equation (Jacopo Riccati, 1676-1754). Furthermore, applying the baker transform of Riccati equation we derive the Einstein thermal diffusion equation governing neuron's transmitting ion's dynamics in the propagation wave front. Given the aforementioned background, we review the neurophysiology that the most abundant cells in our brains are glia (Greek: glue), which are ten time smaller but ten times more abundant than the $\mathrm{mm}$ size neurons. Their functionalities are more than passive house-keeping cleaning brain debris at the nights avoiding dementia Alzheimer disease, but also actively modulate the ion diffusion firing rates during the days. Especially, the glia cells generate a derivative of cholesterol fatty acids known as Myelin sheath coating of the Axon to keep Calcium ionic current within, without being short circuit with the other causing Epileptic seizures. The insulation is sausage-like modulation from the root reservoir Axon Hillock all the way to reach the other neuron's Dendrite Tree. The longest Axon is over a meter long from the head to the toe. While one ion is push inward from the Axon Hillock, the other end ion will be out to the Dendrite in a real time. That's how we issue the command to run away from predators, e.g. Tiger, or chase after preys. A temporary pile up of Calcium ions at the Axon Hillock is possible in the sense of increasing the concentration in backward propagation. This temporarily out of the normal order of forward propagation might be responsible for the bifurcation leading to chaotic dynamics of Walter Freeman, as well as the envelope of bifurcation maps becomes Lotfi Zadeh fuzzy membership function.

We postulate such a sigmoid- $\sigma$ logic augmented with an extra piece of negative logic can generate single hump Feigenbaum-like iterative bifurcations. The envelop function of bifurcation map resembles the Fuzzy Membership Function. Now that we have derived the FMF out of a piece-wise Chaotic brain, we can apply a set of FMF to endow $\mathrm{AV}$ with the understanding pedestrians crossing a red light as a possibility fuzzy thinking in a half the time needed as the dozen years predicted by Highway Traffic Security Agency without FMF's.
\end{abstract}

Keywords: neuronal firing, Calcium, dendrite tree, ion diffusion, chaotic brain
Volume 2 Issue 3 - 2018

\author{
Harold Szu,' Takeshi Yamakawa² \\ 'Department of Biomedical Engineering, The Catholic University, \\ USA \\ ${ }^{2}$ Fuzzy Logic System Institute, Japan
}

\begin{abstract}
Correspondence: Harold Szu, Department of Biomedical Engineering, The Catholic University, Wash DC, USA, Tel 2404 8268 89; Email szuharoldh@gmail.com
\end{abstract}

\section{Acknowledgements}

ONR Grant Award Number N000 I 4- I 7-I-2597.

Received: May 01, 2018 | Published: May 09, 2018
Abbreviations: ANN, artificial neural networks; AV, autonomous vehicle; FMF, fuzzy membership functions; AI, artificial intelligence

\section{Introduction}

Takeshi Yamakawa has successfully applied fuzzy logic to consumer electronics and made the sale in Japan, because the word "fuzzy" sounds like the famous "Fuji" mountain in Japan. For example, Japan has fuzzy air conditioning, fuzzy coffee pot, fuzzy cloth washer, fuzzy automatic transmission, except fuzzy camera which might not sell in the World. When the $3^{\text {rd }}$ Gen AI has incorporated the Lotfi Zade's fuzzy membership functions (FMF) capturing vehicle dynamics, and pedestrian emotional responses, we believe Autonomous Vehicle (AV) called fuzzy Toyota may take off. We refer to Google-developed Alpha Go Artificial Intelligence (AI) as the $2^{\text {nd }}$ Gen AI that Google applies artificial neural network (ANN) to learn the 'If: Then' rules as ANN's I/O. The $1^{\text {st }}$ Gen AI is a fixed Rule-Based System proposed by Marvin Minsky of MIT circa 1990. The $2^{\text {nd }}$ Gen AI can beat human on the Go-Chess Game in 2015. Yet, the $2^{\text {nd }}$ Gen AI cannot yet help human drive an Autonomous Vehicle (AV), for it unfortunately killed a pedestrian in Phoenix Arizona in 2018, We believe that the $2^{\text {nd }}$ Gen AI did not understand human analog 
possibility thinking. We sometimes violate sensibly the traffic rule during a red traffic light when a human driver will take a right hand turn yielding pedestrians \& cars. However, AV cannot do that. This might be due to the automation computer scientists have not digitally captured the human emotional intelligence and program multi-level fuzzy logic in thinking. When this is digitally done successfully, the $3^{\text {rd }}$ Gen AI machine can exit with human being peacefully.

The Myelin sheath that is the fatty acids protein generated by glia cells to separate and speed the transmission of ion current and protects it from short-circuiting from another cell, e.g. causing Epileptic seizure. The glia cells are dynamic in nature, and they can be temporary out of order but are healthy and can rejuvenate themselves with own cell mitosis of course, some rare cases due to genetic genome or life style epigenetic phenome reasons, glia cell can be developing toward tumor (benign type \& malign glioma type 1 to type 4 according to United Nations, World Health Organization, e.g. Senator John McCain has diagnosed glioma in July 2017 after local surgery, he is rapidly worsen to the terminating stage- 4 by April 2018). Multiple sclerosis is another glia causing autoimmune disease that our own immune cells attack and destroy the Myelin sheath. The immune system might be fighting the infection by a virus mistaking
Myelin sheath for a viral protein because it resembles the previouslyrecognized viral invader. We are describing a healthy brain to causing occasional fuzzy possibility thinking, and definitely not pathological reasons, as this might be proved by founder Lotfi Zadeh who lived healthy \& productive until 98 years old without brain disorders.

\section{Unification theory between normal brian and fuzzy brian}

We wish to unify the human brain biological neural networks (BNN) with the brain isothermal natural intelligent (NI)) with Lotfi Zadeh fuzzy logic and Walter Freeman ion diffusion dynamics. We postulated a homeostasis equilibrium theory of human brains which have two states: input state from the dendrite tree to the output state at axon hillock where the potential drop is derived from the MaxwellBoltzmann ( $2^{\text {nd }} \& 1^{\text {st }}$ from LHS of Figure 1$)$ Canoni al probability. It turned out to yield the normalized sigmoid function ${ }^{\sigma}$ of neuron firing rate. This is first observed in 1943 by Warren S. McCulloch $\left(3^{\text {rd }}\right)$, a neuroscientist, and Walter Pitts $\left(4^{\text {th }}\right)$, a logician, in Figure 1. In this paper McCulloch and Pitts tried to understand how the brain could produce highly complex logic prior to John von Neumann $\left(5^{\text {th }}\right.$ from LHS) designing the computer logic. ${ }^{1-4}$
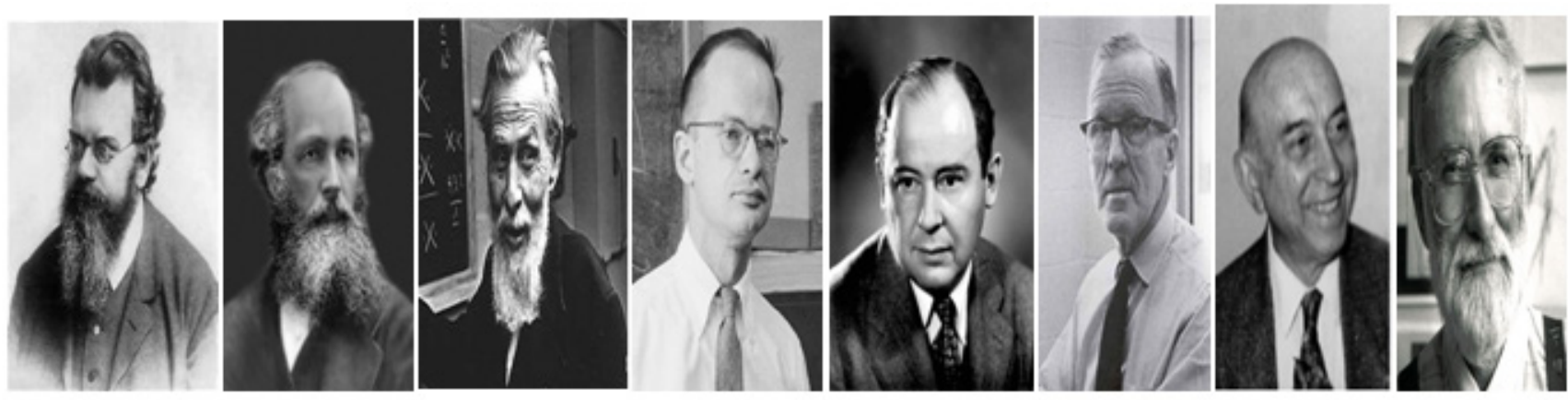

Figure I Neuroscientists contributed human brain dynamics. They are Luwig Boltzmann, James Clerk Maxwell, Warren S. McCulloch (1943) U. Illinois;Walter Pitts (1943) U. Chicago; John von Neumann(1903, 1957) (Donald O. Hebb (1949) U.Toronto., Lotfi Zadeh (98 years old, 2017) UC Berkeley, Walter Freeman (89 years old, 2017), UC Berkeley.

Ludwig Boltzmann introduced the concept of total entropy $S$ as the measuring the degree of uniformity. For example, the mountain top rock has much less entropy than white sand beach

$$
S_{\text {tot }}=k_{B} \log W_{M B},
$$

Where the proportional constant $\boldsymbol{k}_{B}$ has appended with a upper case letter B in honor of Boltzmann. The magnitude of $\boldsymbol{k}_{\boldsymbol{B}}$ is best

represented together with the hot room temperature $27^{\circ} \mathrm{C}$ in terms of the absolute scale of Kelvin Temperature $T$

$$
27^{\circ} \mathrm{C}+273^{\circ} \mathrm{K}=300^{\circ} \mathrm{K}=\left(\frac{1}{40}\right) \mathrm{eV}
$$

$W_{M B}=\exp \left(\frac{S_{\text {tot }}}{k_{B}}\right)=\exp \left(\frac{\left(S_{\text {brain }}+S_{\text {env. }}\right) T_{o}}{k_{B} T_{o}}\right)=\exp \left(\frac{S_{\text {brain }} T_{o}-E_{\text {brain }}}{k_{B} T_{o}}\right)=\exp \left(-\frac{H_{\text {brain }}}{k_{B} T_{o}}\right)$

Thus, human brain temperature is kept at $\mathrm{T}=37{ }^{0} \mathrm{C}$ which is slightly higher than the thermal reservoir energy which is optimum for the elasticity of red blood cell hemoglobin. On the other hand, the chicken is kept at $40{ }^{\circ} \mathrm{C}$ for reason of hatching eggs. However, a higher temperature of brains is not necessarily to be smart, because we "ate chicken, not vice versa. Q.E.D." Maxwell-Boltzmann Probability $\mathrm{W}$ is derived from the third thermodynamic Neal's law at non-zero temperature that insures the incessant collision mixing homogenizing the degree of uniformity measured by the total entropy

$$
S \uparrow=S_{\text {env }}+S\left(x_{0}\right) \uparrow=k_{B} \log \left(W_{M B} \uparrow\right) ; W_{M B}\left(x_{o}\right)=\exp \left(-H_{\text {brain }}\left(x_{o}\right) / k_{B} T_{o}\right),
$$

where $\boldsymbol{H}_{\text {brain }}$ is the derived within the head $\left(\boldsymbol{x}_{0}\right)$ is called the Helmholtz Free Energy $\mathrm{H}_{\text {brain }}\left(\mathrm{x}_{\mathrm{o}}\right)$ defined as the internal energy $\mathrm{E}_{\text {brain }}\left(\mathrm{x}_{\mathrm{o}}\right)$ in contact with a blood environment at the temperature $\boldsymbol{T}_{\boldsymbol{o}}$ .The free energy $\boldsymbol{H}_{\text {brain }}\left(x_{o}\right)$ is the total internal $\boldsymbol{E}_{\text {brain }}\left(x_{o}\right)$ subtracted the thermal entropy energy $\boldsymbol{T} \boldsymbol{S}\left(\boldsymbol{x}_{0}\right)$ and the net becomes free-to-do work energy kept to be the minimum to be stable:

$$
\text { min. } \boldsymbol{H}_{\text {brain }}\left(x_{o}\right) \downarrow=\boldsymbol{E}_{\text {brain }}\left(x_{o}\right)-\boldsymbol{T} \boldsymbol{S}\left(x_{o}\right) \uparrow
$$


Use is made of the isothermal equilibrium of brain in the warm blood reservoir at the homeostasis temperature $T$. Use is further used of the second law of conservation energy $\Delta Q_{e n v .}{ }^{o}=T_{o} \Delta S_{e n v}$ and the brain internal energy. $\Delta E_{\text {brain }}+\Delta Q_{\text {env. }}=0$, and then we integrate the change and dropped the integration constant due to arbitrary probability normalization. We now know $H_{\text {Brain }}$ is related to constant temperature $T_{o}=37 C=310 K$ thermodynamic Helmholtz Free Energy $H_{\text {Brain }}=E_{\text {Brain }}-T_{o} \mathrm{~S}$ (Figure 2).

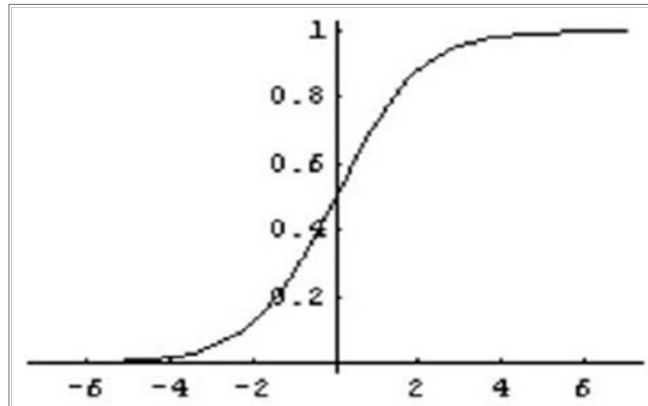

(a)

Figure 2 Standard McCullough-Pittl Sigmoid Threshold Logic is Eq.(5) derived from two state normalization of Maxwell-Boltzmann distribution function Eq.( I).

$\exp \left(-\frac{H_{1}}{k_{B} T_{o}}\right) / \exp \left(-\frac{H_{1}}{k_{B} T_{o}}\right)+\exp \left(-\frac{H_{2}}{k_{B} T_{o}}\right)=1 /\left[\exp \left(\frac{\Delta \mathrm{H}_{1,2}}{k_{B} T_{o}}\right)+1\right]=\sigma\left(\frac{\Delta \mathrm{H}_{1,2}}{k_{B} T_{o}}\right)=\left\{\begin{array}{l}1, \frac{\Delta \mathrm{H}_{1,2}}{k_{B} T_{o}} \rightarrow \infty \\ 0, \frac{\Delta \mathrm{H}_{1,2} \rightarrow-\infty}{k_{B} T_{o}} \rightarrow-\infty\end{array}\right.$

Theorem 1: Riccati nonlinear $1^{\text {st }}$ order differential equation is derived from Maxwell-Boltzman two state weighted sum and its exact solution turns out to be the sigmoid threshold function $\sigma(x)$ : Let $x=\frac{\Delta \mathrm{H}_{1,2}}{k_{B} T_{o}}$

$$
\frac{d \sigma(x)}{d x}+\sigma(x)=\sigma(x)^{2} ; \sigma(x)=\frac{1}{\exp (x)+1}
$$

Proof: $\frac{d \sigma(x)}{d x}=\frac{d}{d x}[\exp (x)+1]^{-1}=-1[\exp (x)+1]^{-2} \exp (x)=-1[\exp (x)+1]^{-2}\{-1+(\exp (x)+1)\}=\sigma(x)^{2}-\sigma(X)$

Theorem 2: Hopf (baker) Transform can linearize the first order Ricati quadratic-nonlinear differential equation to A. Einstein diffusion equation $\mathrm{Eq}(4)$. (Note that in dynamical systems theory, the baker's map is a chaotic map from the unit square into itself. It is named after a kneading operation that bakers apply to dough: the dough is cut in half, and the two halves are stacked on one another, and compressed. cf. Wikipedia)

\section{Proof:}

We introduce the calcium ions $\varphi(x)$ concentration; we can set the slope of logarithmic concentration to be two-state normalization sigmoid

$$
\begin{aligned}
& \sigma(x)=-\frac{\varphi(x)^{\prime}}{\varphi(x)}=-\frac{d}{d x} \log \varphi(x) \\
& L H S=\frac{d \sigma(x)}{d x}=-\frac{\varphi^{\prime \prime}}{\varphi}+\frac{\left(\varphi^{\prime}\right)^{2}}{\varphi^{2}}=R H S=\frac{\left(\varphi^{\prime}\right)^{2}}{\varphi^{2}}+\frac{\varphi(x)^{\prime}}{\varphi(x)} \\
& \varphi^{\prime}=-\varphi^{\prime \prime}
\end{aligned}
$$

With respect to a local wave front, the streaming term is set to zero at the wave front, e.g. sitting on the smoke outer most wave front Figure 3 where smoke particles will be diffusive.

$$
\frac{d \varphi}{d t}=\varphi_{t}+\varphi^{\prime}=0 ; \Rightarrow \varphi^{\prime} \cong-\varphi_{t}
$$

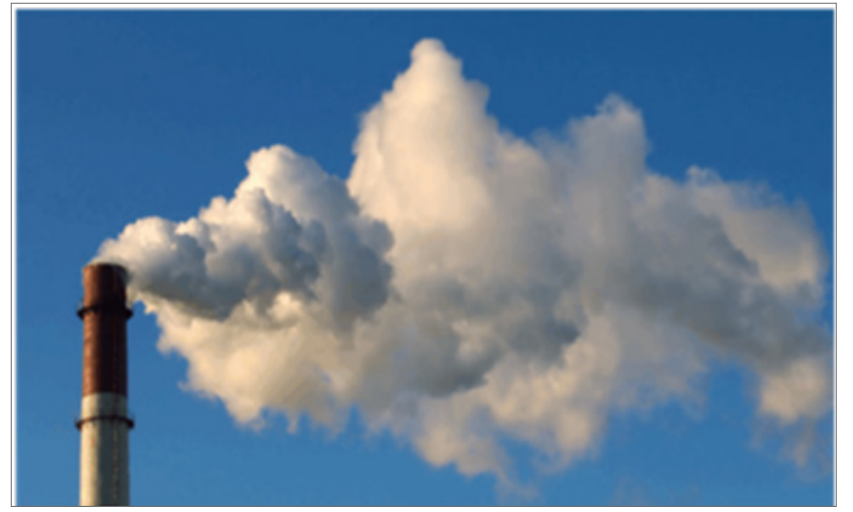

Figure 3 Smoke from chimney is similar to Calcium ions passing from dendrite tree synaptic gaps to axon.

We have derived that at the local wave front of the neuro. transmittent calcium ions the diffusion equation of calcium ions concentration $\varphi(x)$ satisfies Albert Einstein's Brownian motion:

$$
\varphi_{t}=\varphi^{\prime \prime}
$$

From which follows the standard correlation function of the diffusion density by means of Taylor series expansion:

$$
\left\langle\varphi\left(t_{o}\right) \varphi\left(t_{o}+t\right)\right\rangle \cong\left\langle\varphi ( V \times t _ { o } ) \left( V \times\left(t_{o}+t\right)>\cong\left\langle\mathrm{r}^{2}\right\rangle+\frac{1}{2} \varphi_{t_{o}}^{\prime}\langle\mathrm{r}\rangle+\ldots,\right.\right.
$$

Where $V t=r$ the quadratic parabolic curvature is reduced through the collisions among Calcium ion particles and cellar medium molecules into a linear trajectory (Figure 4). However, when the active ushering glia cells are temporarily out of order, these ions cannot keep up with the ions passing rate, the output rate my dip in piecewise negative slope: the more inputs are, the less output are. The ions are stuck in the Axon Hillock (Figure 5).

The quadratic sigmoid map follows M. Feigenbaum bifurcation logistic map with $4 \lambda$ knob turning higher value that might depend on the physiologic happy Hormone Dopamine or glia cells cooperation or not (Figure 6).

$$
\begin{gathered}
y_{n+1}=4 \lambda \boldsymbol{x}_{\boldsymbol{n}}\left(1-\boldsymbol{x}_{\boldsymbol{n}}\right) ; n=1,2,3, \ldots \\
\boldsymbol{x}_{\boldsymbol{n}+1}=\boldsymbol{y}_{\boldsymbol{n}+1}
\end{gathered}
$$


Physiology experiments: Neurobiologists have observed the sudden onset of Epileptic seizure when the two states are crossed over becoming a pseudo-synchronous. The root cause could be the crossover output axon without insulating Myelin sheath due to Epileptic seizures the output short circuit the input dendrite tree in Figure 4B. Multiple sclerosis is another glia causing autoimmune disease that our own immune cells attack and destroy the Myelin sheath. In these cases, Takeshi Yamakawa has demonstrated by using real-time Magnetic imaging the physician can apply a laser pipe in to burn at the cross-over junction. The less invasive procedure has turned the Hospital week-long patients into a day visit out-patient (Figure 7) (Figure 8).

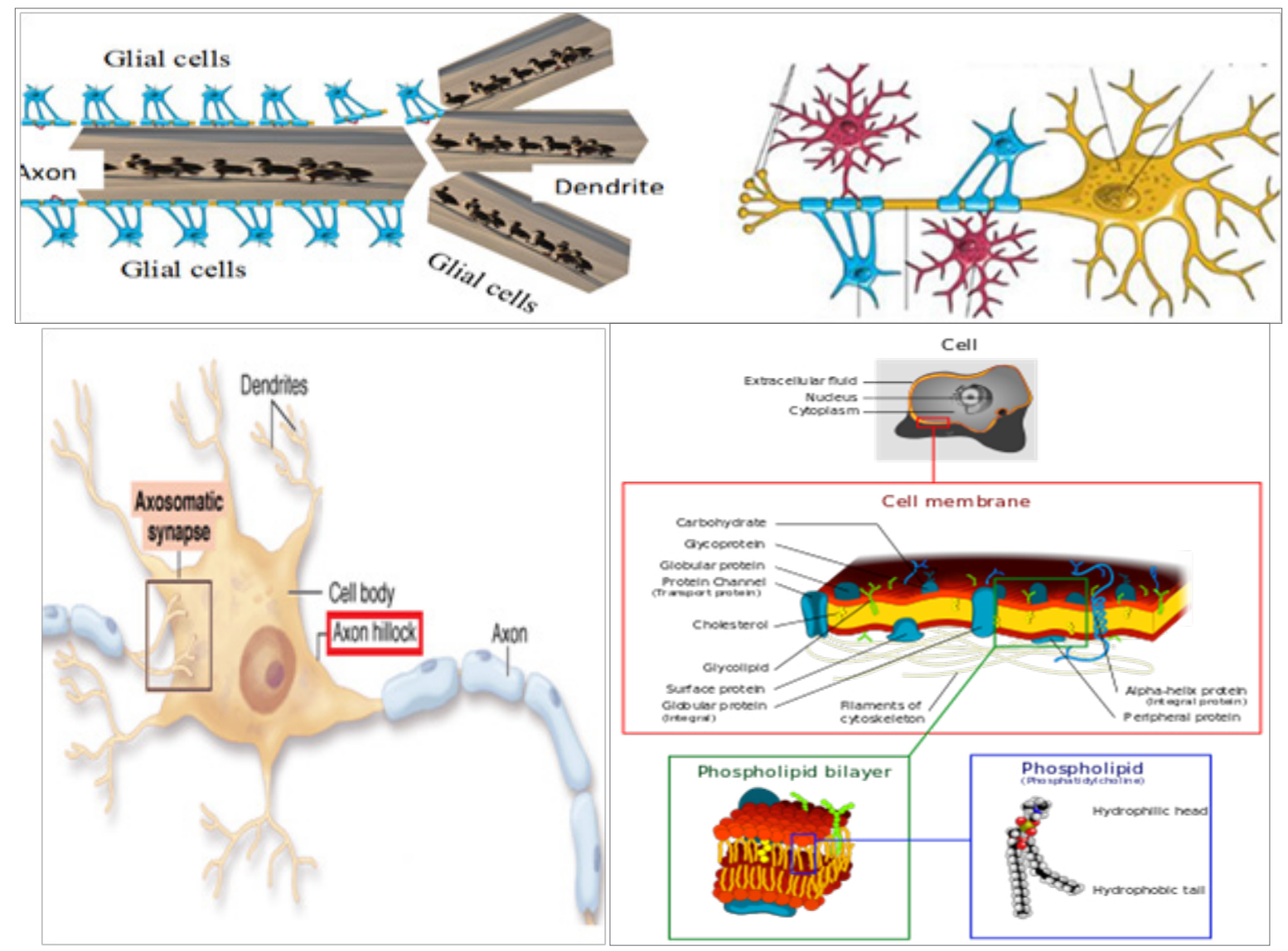

Figure 4 Indicate the complexity of ion current reservoir that (Wikipedia) might go wrong.A single layer of the membrane is built with hydrophilic heads and hydrophobic tails (like a human heads and legs) in three inter-related inserts in the RHS. The potential at the Axon Hillock (LHS sub-figure) has about $~ 100-200$ voltage-gated sodium channels per square micrometer. Both inhibitory postsynaptic potentials and excitatory postsynaptic potentials are summed in the axon hillock and once a triggering threshold is exceeded, an action potential propagates through the rest of the axon (and "backwards" towards the dendrites as seen in neural back-propagation).

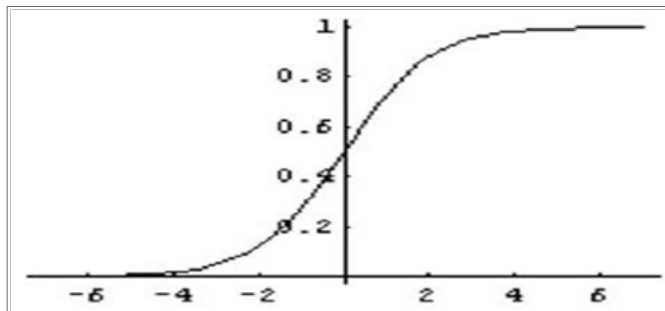

(a)

Figure 5 Chaotic Neural Net will be due to a piecewise negative N-shape logic in the sigmoid logic.

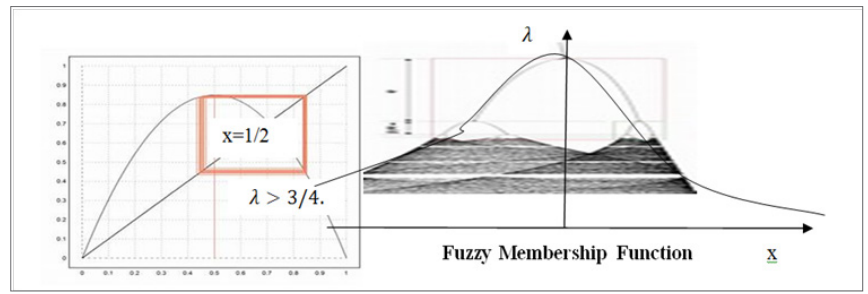

Figure 6 Logistic map demonstrated the bifurcation cascades by means of increasing the knot $\lambda>\frac{3}{4}$ so that the slope of hump at the intersecting the feedback line at $45^{\circ}$ becomes larger than $90^{\circ}$ (Michele Feigenbaum, circa 1970). 


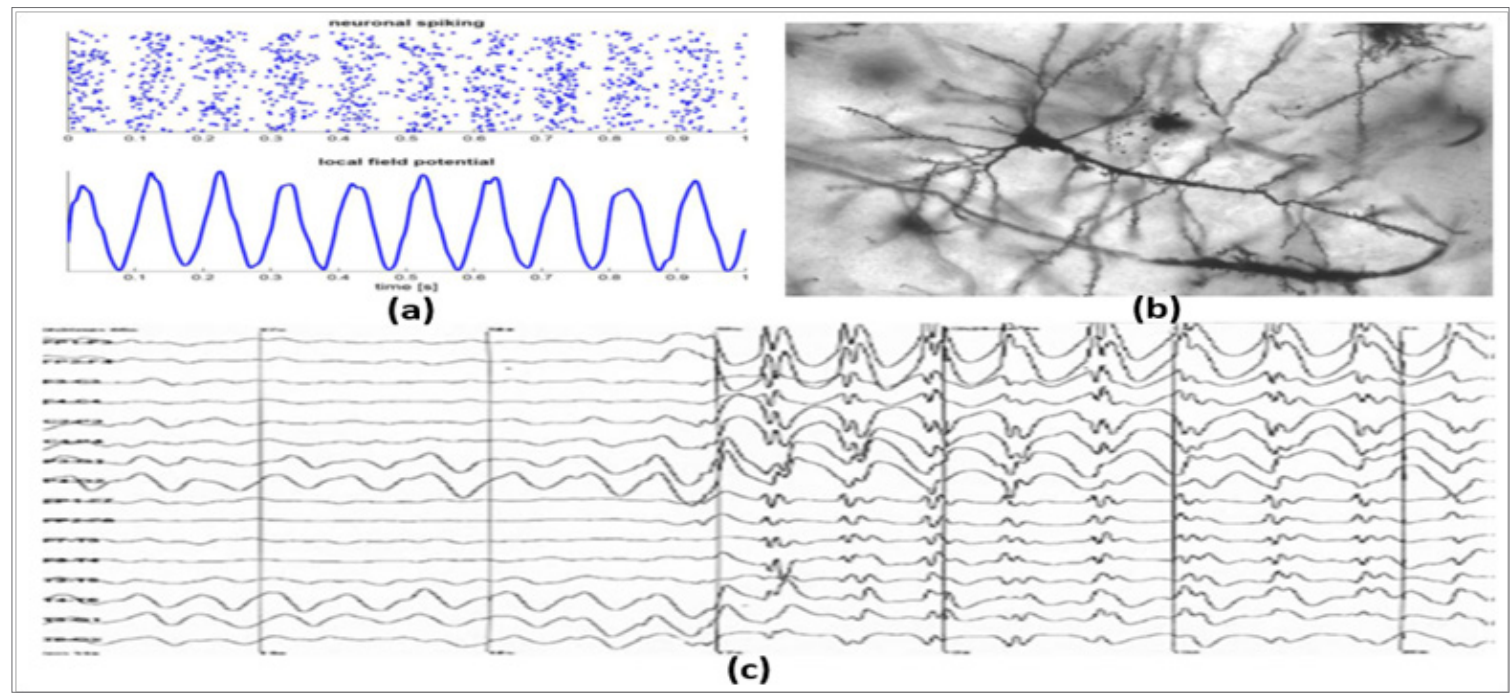

Figure 7 Fundamentally, we can understand the neuron density modulation of firing rates by means of Donald O. Hebb phenomenological rule: "linked together, firing together"(LTFT), (observed 5 decade ago by Hebb in U. Toronto) that is why the dots density appears to be modulated from on I00 Hz to off less than $50 \mathrm{~Hz}$. In reality, there is no travelling Electromagnetic wave in the BNN Figure 4 (a), rather neuronal population of firing rates.
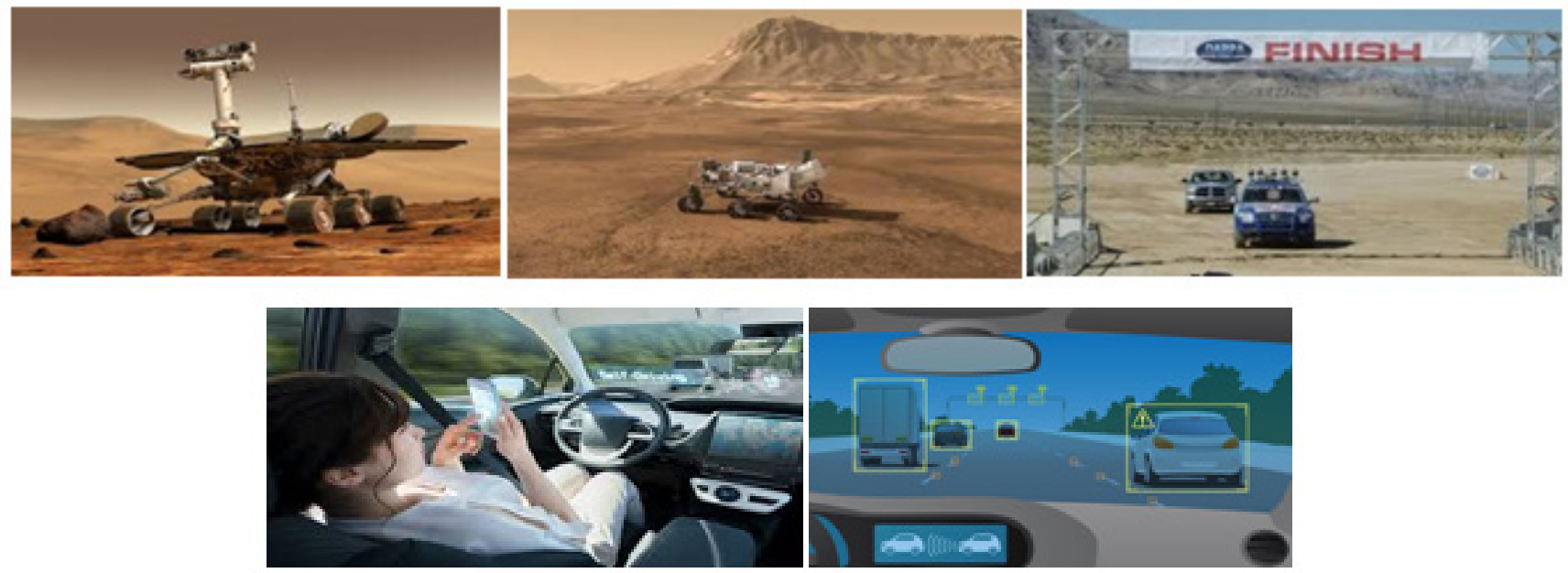

Figure $8 \mathrm{~A}$ long history of NASA and DARPA have invested on Driverless Autonomous Vehicles (DAV). Nevertheless, in no-man land, the scientists \& technologists have not taken into account human driver and pedestrian behaviors. Applying Al to Sensors Suites is both all-weather W band Radar and optical LIDAR, as well as the Video Motion Detection Optical.

\section{Applications to Autonomous Vehicle (AV)}

Flow Imaging Processing is adopted for collision avoidance situation awareness.

Why driverless autonomous vehicle (DAV) takes dozen years (according to Science Magazine (V. 358, pp.1370-1375, Dec 15, 2018)? This might be due to that recently Uber's DAV has killed a pedestrian in Phoenix Arizona. When can Human Experience Expert Systems be coded as the $3 \mathrm{rd}$ Gen AI that can understand and co-exist with Human? We notice that a major difference between machines versus human is that digital closed set table of look up versus analog open set agenda. For example," a traffic rule of red light is made to be sensibly broken"; they shall do no harm to the other. The $1^{\text {st }}$ Gen AI "Rule-Based System," M. Minsky when F. Rosenblatt built visions failed. The $2^{\text {nd }}$ Gen AI "Learnable rule-based" has beaten Lee Sedol in 4:1 Mar.19, 2015. The $3^{\text {rd }}$ Gen AI "Human Experience Expert System" Google, Uber \& Tesla in US, German, Italy, and Japan have spent over \$100B to DAV's. Recently a DAV killed a pedestrian in Phoenix Arizona. In spite of NASA Space Program and DARPA Grand Challenge, technologies were in no-man land. Highway TSA said semi-DAV at level 4 will be dozen years, circa 2030. DAV machine must be peacefully co-exited with crowded human society with all kinds of personality. Automation computer scientists need to endow machines with $3^{\text {rd }}$ Gen AI to comprehend human fuzzy possibility thinking, so-called Zedah-Freeman fuzzy logic, in order to exit with human being society. Of course, fuzziness is not the logic, but open set Fuzzy Membership Function's (FMF's)) e.g." "Young", or "Beautiful." A double fuzziness "Young \& Beautiful" becomes less fuzzy. That's why we endow FMF's at execution load time to the digital machine. ${ }^{5-8}$ 
A. Statistical Ergodicity principle allows us to replace the limited temporal average with massively parallel spatial averages to accommodate all possible initial boundary conditions,

B. Biological Natural Intelligence base on aforementioned homeostasis principle, we have derived the Biological Neural Nets sigmoid logic from which we have reproduced Walter Freeman diffusion and 1Otfi Zadeh fuzzy logic membership function.

C. Human psychology \& fuzzy thinking about the traffic rules. We shall code the fuzzy logic into machine's Control Command and
Communication Intelligence(C4I), but FMF's are sharpen after Boolean Logic $(U, \cap)$ at load time. Model \& Simulate $(M \& S)$ each dynamics including (1) Hebb Learning (2) road-friction LangevinEinstein, (3) Satellites-GPU, (4) Computer Sensing (Radar, Lidar, Video, Sound, Tactile, Smell, Sensors).

D. Multiple Layer known Deep Learning ${ }^{4,9}$

E. To increase probability of detection of A \& minimize False Alarm Rate B we need multiple layers in the so-called deep learning (Figure 9).
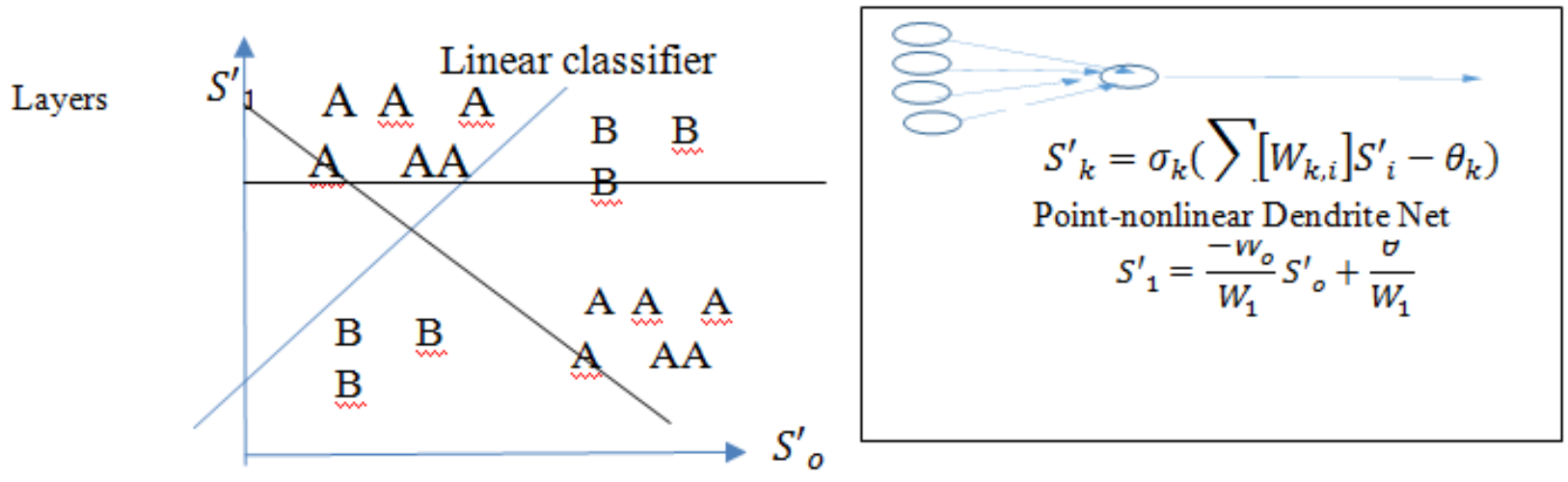

Figure 9 Artificial Neural Networks (ANN) needs multiple layers known as Deep Learning 8 (a) Left panel shows that while a single layer of Artificial Neural Network can simply be a linear classifier, the right panel shows the dendrite net giving rise to the linear classifier equation after the sigmoid threshold.

We wish to improve the supervised deep learning with LMS cost to become self-organization "follow the leader" as Unsupervised Deep Learning with thermodynamic equilibrium at constant temperature $T$ at Minimum Free Energy (MFE). The automation solves NP Complete path finding problem by means of loss-less divide and conquer (ANN TSP, Foo \& Szu, 1997). Supervised cost function is taken as $\min \|\vec{A}-\vec{B}\|=\min \|\vec{A}-\vec{C}+\vec{C}-\vec{B}\|=\min \|\vec{A}-\vec{C}\|+\min \|\vec{C}-\vec{B}\|$ iff $\perp$ , the cost of search for $\vec{C}$ city is linear at boundary. We review thermodynamics to answer why keep our head blood temperature constant $37^{\circ} \mathrm{C}$. This is necessary for the optimum elasticity of red blood cells Hemoglobium, squeezing through capillaries supplying the glucose and oxygen. Also, the thermodynamic equilibrium keeps the chemical diffusion rate constant for all generations to accumulate the experience. This is the basis of human Natural Intelligence (NI) based on two necessary conditions:

I. Constant temperature brain and (ii) power of pairs.

II. Applying Least Mean Squares (LMS) Error Energy,

$$
E=\mid\left(\text { desired } \text { Output } \vec{S}_{\text {pairs }}-\text { actural Output }\left.\hat{S}_{\text {pairs }}(t)\right|^{2}\right.
$$

Sensory Inputs: "While agreed, the signal; disagreed, the noises"

$$
\text { Power of pairs: } \vec{X}_{\text {pairs }}(t)=\left[A_{i j}\right] \vec{S}_{\text {pairs }}(t)
$$

The agreed signals become the vector pair time series $\vec{X}_{\text {pairs }}(t)$ with the internal representation of degree of uniformity of neirs firing rate $\vec{S}_{\text {pairs }}(t)$ described with Ludwig Boltzmann entropy with unknown space-variant impulse response functions mixing matrix $\left[A_{i j}\right]$ and the invers by learning synaptic weight matrix.

\section{The inverse is convolution artificial neural networks}

$$
\hat{S}_{\text {pairs }}(t)=\left[W_{j i}(t)\right] \vec{X}_{\text {pairs }}(t)
$$

Subtracted the un-usable thermal noise energy $T_{0} S$

$$
\begin{aligned}
& H \downarrow=E-T_{O} S \uparrow \geq 0 \\
& \frac{d[W]}{d t}=-\frac{\partial H}{\partial[W]}
\end{aligned}
$$

Control steering wheel Lyaponov convergence of Learning of

$$
\frac{d H}{d t}=\frac{\partial H}{\partial[W]} \frac{d[W]}{d t}=\frac{\partial H}{\partial[W]}\left(-\frac{\partial H}{\partial[W]}\right)=-\left(\frac{\partial H}{\partial[W]}\right)^{2} \leq 0
$$

Langevin equation of the car momentum $\vec{P}=m \vec{V}$, with tire-road friction coefficient $f$,car-body aerodynamic fluctuation force $\vec{F}(t)$

$$
\frac{d \vec{P}}{d t}=-f \vec{P}+\vec{F}(t)
$$

$$
\left\langle\vec{F}(t) \cdot \vec{F}\left(t^{\prime}\right)\right\rangle=2 k_{B} f \delta\left(t-t^{\prime}\right)
$$

This possible membership concept is important to exploration of large data as which often don't have definitive membership relations when partial analysis of the data is being done without definite knowledge that classifies all the subsets of the data. For example, "young and beautiful" is a much sharper possibility than either "the 
Young" or "the Beautiful". When we average over spatial cases, we obtain the average of the experience based Expert System in order to elucidate $i-A I$.

Brake FMF $\cap$ Sensor Awareness FMF $\cap$ GPS space - time FMF $=$ Experience $\sigma($ stop $)$

Review of fuzzy membership function, which is an open set and cannot be normalized as the probability but a possibility (Figure 10). UC Berkeley Prof. Lotfi Zadeh passed away at the age of 95 years old and Walter Freeman at age 89 . To them, 80 may be "young." Likewise, the "beauty" is in the eye of beholder. According to the Greek mythology of Helen of Troy, has sunk thousand ships, and Egypt Cleopatra hundred ships and Bible Eva one ship (Noah Arc) (Figure 11). Consequently, the car will drive through slowly when the red light happens at the mid night in desert and without incoming cars. Such an RB becomes flexible as EBES. To show that this replacing $\boldsymbol{R} \boldsymbol{B}$ with $\boldsymbol{E} \boldsymbol{B} \boldsymbol{E} \boldsymbol{S}$ is a natural improvement of $A I$ in the remaining paper. This explains a driverless car that will turn the rule stopping at red light to be gliding over the red light when no incoming car at mid light in desert. ${ }^{10-16}$

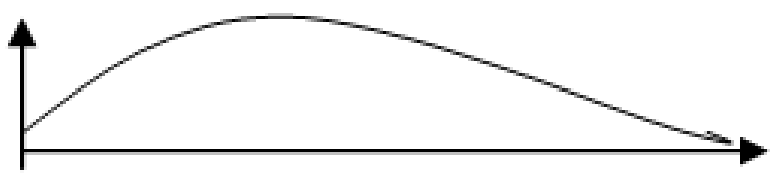

Figure I 0 Young membership is not well defined:Young (I7 to 65).

\section{Conclusion}

We demonstrate an application of Lotfi Zadeh fuzzy membership function of two states "beauty or not" (Figure 12). In summary, Russian Mathematician G Cybenko ${ }^{2}$ has proved "Approximation by Superposition of a Sigmoidal Functions," Math. Control Signals Sys. (1989)2:303-314. Similarly, Kolmogorov $\mathrm{AN}^{3}$ has given "On the representation of continuous functions of many

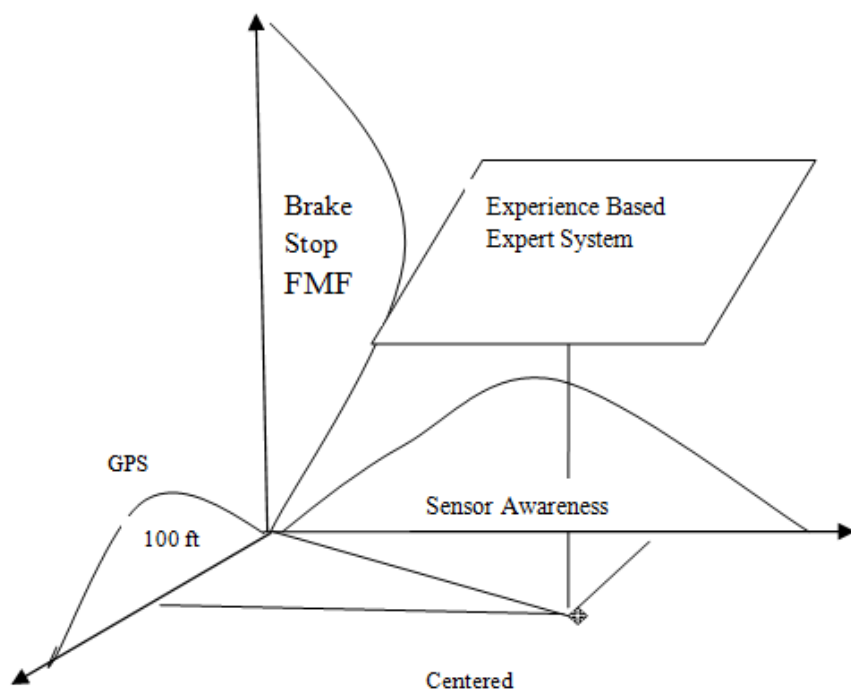

Figure I I The utility of FMF logic is Boolean Logic of Union $U$ \& Intersection $\cap$ of open set Fuzzy Membership Functions (FMF) which cannot be normalized as the probability. The Boolean logic is sharp, not fuzzy. Unfortunately, the shortened "Fuzzy (membership function) Logic" as "Fuzzy Logic" is a misnomer Logic cannot be fuzzy, but the set can be open set as all possibilities. Szu has advocated a bifurcation of chaos (advocated first by Walter Freeman in human brains with Bob Kozma) as a learnable FMF, making the deterministic Chaos as the learnable dynamics of FMF (cf. Max Planck: ResearcGate.net).

variables by superposition of continuous function of one variable and addition,'Dokl. Akad. Nauk, SSSR, 114(1957), 953-956. The two state normalization in the Maxwell-Boltzmann phase space distributions is derived to be equivalent to an ion-current diffusion equation, as proposed first ad-hoc-ly by Walter Freeman. By means of the Hopf transform, we can be applied to the sigmoid threshold logic, which turns out to be fuzzy membership function (FMF) of beauty or not. We have illustrated the two states normalization.

\section{Fuzzy Logic: e.g. Beauty is in the eye of beholder:}

Adam and Eva(1 ship Noah Ark), Egypt Cleopatra (sunk 10 ships); China Xi Shi (西施) (sunk 100 birds \& fishes); Helen of Troy (sunk 1000 ships); your sweet heart might be in the limit of infinite ships (the phase transition of infinity ships could wreck the Scholarship)

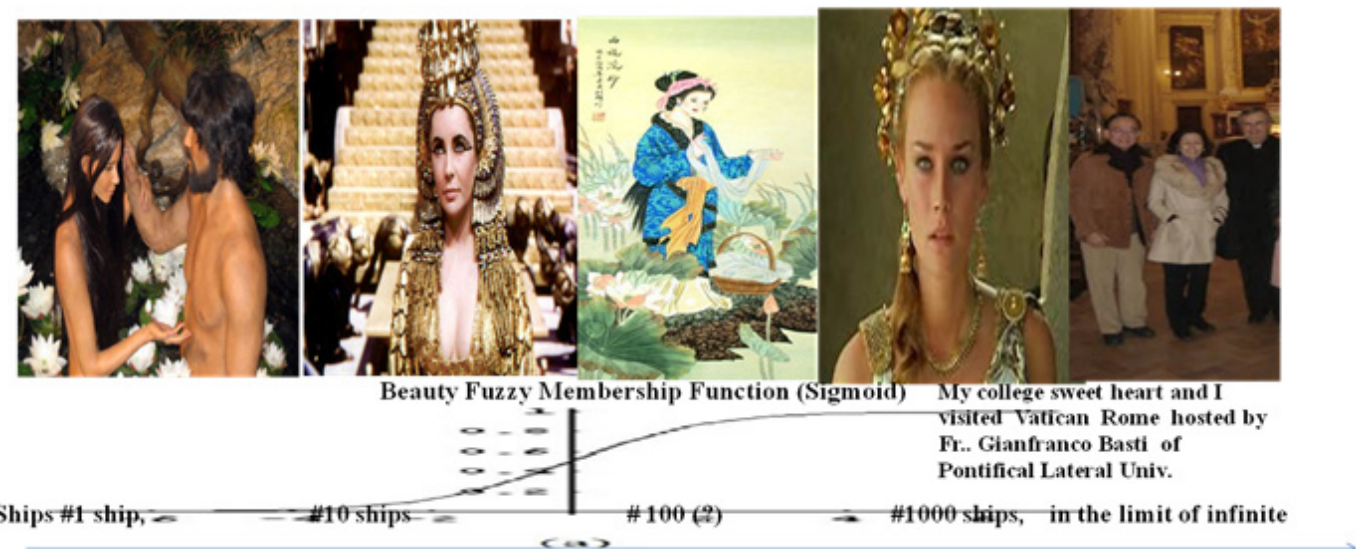

Figure I 2 Beauty Fuzzy Logic Membership Function turns out to sigmoid. Since the beauty is in the eyes of beholder, then it follows two state beauty or not in terms of Maxwell-Boltzmann phase space distribution and derived the sigmoid function, Eq.(5). 


\section{Conflict of interest}

Authors declare that there is no conflict of interest.

\section{References}

1. Warren S, McCulloch, Walter Pitts. A logical calculus of the ideas immanent in nervous activity. The Bulletin of Mathematical Biophysics. 1943;5(4):115-1339.

2. Cybenko G. Approximation by Superposition of a Sigmoid Functions Math Control Signals Sys. 1989;2:303-314.

3. Kolmogorov N. On the representation of continuous functions of many variables by superposition of continuous function of one variable and addition. Dokl Akad Nauk SSSR. 1957;114(5):953-956.

4. Donald O Hebb. The Organization of Behavior: A Neuropsychological theory. New York: Wiley and Sons; 1949.

5. DARPA call for explainable AI or XAI Website, 2017-2020 \$10M.

6. ONR call 6.1 AI proposal website, 2018-2021 \$10M.

7. We know there're out there. New Scientist Weekly March. 2016:19-25.
8. When will we get there" Science V. 358, 15 Dec 2017.

9. Hinton G, Lecun Y, Bengio Y. Deep learning. Nature. 2015.

10. James McCelland, David Rumelhart. Backprop. Book MIT Press; 1986. p. 1 .

11. Soo-Young Lee, Harold Szu. Design of Smartphone Capturing Subtle Emotional Behavior. MOJ App Bio Biomech. 2017;1(2):1-10.

12. Lipmann RK. Introduction to computing with neural nets. IEEE ASSP Magazine. 1987.

13. Harold Szu, Mike Wardlaw, Jeff Willey, et al. Theory of glial cells \& neurons emulating biological neural networks (bnn) for natural intelligence (ni) operated effortlessly at a minimum free energy (mfe). MOJ App Bio BioMech. 2017;1(1):1-26.

14. Harold Szu, Gyu Moon. How to avoid DAD? MOJ App Bio BioMech. 2018;2(2):43-58.

15. http://ica-wavelet.net

16. Ronald J Fox. Construction of the Jordan basis for the Baker map. Chaos; 1997;7:254 